\title{
Identifying Self-reported Health-related Problems in Home-based Rehabilitation of Older Patients after Hip Replacement in China: A Machine Learning Study based on Omaha System Theory
}

\section{Jing Chen}

Shanghai Tenth People's Hospital, School of Medicine, Tongji University, Shanghai, China

\section{Fan He}

Smart Engineering Research Institute, Shanghai Investigation, Design \& Research Institute Co.,Ltd., Shanghai, China

\section{Qian Wu}

Shanghai Tenth People's Hospital, School of Medicine, Tongji University, Shanghai, China

\section{Li Wang}

Shanghai Tenth People's Hospital, School of Medicine, Tongji University, Shanghai, China

\section{Xiaoxia Zhu}

Orthopaedics Department, Changhai Hospital, the Second Military Medical University, Shanghai, China

Yan Qi

School of Medicine, Jinggangshan University, Ji An, China

\section{Yan Shi ( $\nabla$ nih0325@163.com)}

Shanghai Tenth People's Hospital, School of Medicine, Tongji University, Shanghai, China

\section{Research Article}

Keywords: hip replacement, home-based rehabilitation, machine learning, older individual, self-reported outcome

Posted Date: February 1st, 2021

DOl: https://doi.org/10.21203/rs.3.rs-154299/v1

License: (c) (i) This work is licensed under a Creative Commons Attribution 4.0 International License. Read Full License 
Identifying self-reported health-related problems in home-based rehabilitation of older patients after hip replacement in China: A machine learning study based on Omaha system theory

Jing Chen ${ }^{1^{*}}$, Fan $\mathrm{He}^{2}$, Qian Wu ${ }^{1}$, Li Wang ${ }^{1}$, Xiaoxia $\mathrm{Zhu}^{3}$, Yan $\mathrm{Qi}^{4}$, Yan Shi ${ }^{{ }^{*}}$

\section{Corresponding author:}

*Correspondence: tegobao@163.com;*nih0325@163.com

1 Shanghai Tenth People's Hospital, School of Medicine, Tongji University, Shanghai, China Full list of author information is available at the end of the article

Keywords: hip replacement, home-based rehabilitation, machine learning, older individual, self-reported outcome 


\section{Abstract}

Background: With the aging of the population, the number of total hip replacement surgeries is increasing globally. Hip replacement has undergone revolutionary advancements in surgical methods and materials. Due to the short length of hospitalization, rehabilitation care is mainly home-based. The needs and concerns about such home-based rehabilitation are constantly changing, requiring continuous attention.

Objective: To explore effective methods for comprehensively identifying older patients' self-reported outcomes after home-based rehabilitation for hip replacement, in order to develop appropriate intervention strategies for patient rehabilitation care in the future.

Methods: This study constructed a corpus of patients' self-reported rehabilitation care problems after hip replacement, based on the Omaha classification system. This study used the Python development language and implemented artificial intelligence to match the corpus data on the cooperation platform, to identify the main health-related problems reported by the patients, and to perform statistical analyses.

Results: Most patients had physical health-related problems. More than $80 \%$ of these problems were related to neuromusculoskeletal function, interpersonal relationships, pain, health care supervision, physical activity, vision, nutrition, and residential environment. The most common period in which patients' self-reported problems arose was 6 months post-surgery. The relevant labels that were moderately related to these problems were: Physiology—Speech and Language and Physiology-Mind $(r=0.45)$, Health-Related Behaviors-Nutrition and Health-Related Behaviors - Compliance with Doctors' Prescription $(\mathrm{r}=0.40)$. 
Conclusion: Physiological issues remain the main health-related issues for home-based rehabilitation after hip replacement in older patients. Precision care has become an important principle of rehabilitation care. This study used big data law to obtain the largest quantitative network data possible. The artificial intelligence capture was fully automated, which greatly improved efficiency, as compared to manual data entering.

Keywords: hip replacement, home-based rehabilitation, machine learning, older individual, self-reported outcome 


\section{Introduction}

In terms of population aging, China is in the rapid development stage of aging, with concomitant medical problems [1]. In older individuals, the reduction in total bone mass facilitates femoral fractures [2]. According to 5-year (2008-2012) statistics from Beijing Jishuitan Hospital [3], there is an average annual increase of $7.3 \%$ in patients older than 50 years with femoral neck fracture in China. Hip replacement surgery is the most commonly used treatment for this condition, and effectively relieves pain, improves limb function, and enhances the patient's quality of life, but postoperative rehabilitation influences the long-term outcomes. Due to the short length of hospital stay of such patients, rehabilitation is mainly home-based [4].

In recent years, hip replacement has undergone advances in surgical methods and materials, and the focus of care has widened. The needs and concerns about patients' home-based rehabilitation are also continuously changing. Thus, it is crucial to identify and pay attention to issues that have gone unattended in a timely and continuous manner, to consider whether the content of rehabilitation interventions currently provided is sufficient, and to make careful efforts to perform appropriate interventions from the patient's perspective.

The current methods for surveying home-based rehabilitation patients, particularly for long-term follow-up, are often manpower- and material resource-intensive. If the patient recovers well after surgery, follow-up may be regarded as a burden and even as unnecessary [5]. Thus, it is necessary to weigh up the benefits and drawbacks of rehabilitation, and to consider better means of follow-up and data collection. With the increase in big data, 
scientific research methods can overcome various obstacles to the assessment of home-care. New methods can identify the main problems and needs in home-based rehabilitation more efficiently, based on patients' self-reported outcomes [6]. For instance, the content published on Twitter and other social media by users with related diseases have been studied to explore the emotional experience and needs of patients in the real world [7], and could also be applied to home-based rehabilitation research. Given this context, the present study sought to use machine learning to perform data mining and analysis of big data using such network resources.

In this study, an online consultation platform, involving WeChat and the Worldwide Web, directly related to the study was selected as the data source. Using this online consultation platform directly related to the study hospital, patients who were discharged home and needed online help from medical staff posed questions to doctors, using smart devices or computers, and doctors then answer these questions online. The platform hides patients' personal information, and users can choose the content to be disclosed on the communication platform. To facilitate comparisons of our findings with other studies, we selected the Omaha system to guide data classification. It is an effective tool for collecting, sorting, recording, and analyzing patient data in clinical work [8-10]. Using this theory, we constructed a lexicon for matching keywords to analyze the main problems experienced by older patients undergoing home-based rehabilitation after hip replacement as self-reported on the online platform. 


\section{Methods}

We first obtained a historical sample set from the platform and carried out text analysis on the questions raised by patients. After literature review and manual analysis of patients' descriptions, an expert team used the Delphi method to construct a third-party lexicon (A Keyword Lexicon of Patients' Self-reported Rehabilitation Care Problems after Hip Replacement), and then statistically analyzed the sample set based on the lexicon, using supervised machine learning, to identify and rank the main problems patients experienced, and to analyze the correlations between problems.

\subsection{Data source}

After obtaining permission from the platform, we selected all information on patients' online consultation with orthopedic doctors on the Good Doctor Online platform that cooperated with our hospital, and used Python 3.6 to write a crawler program to perform structured processing of this text, based on the Scrapy crawler framework. The data were divided into 14 pieces of fine-grained information. The steps were as follows: (1) The URL, title, and content of each consultation content were extracted. (2) The content was further broken up into fine-grained information pieces according to 12 tags: disease, examination data, imaging data, description of the condition, medication status, length of illness, hospital and department visited, previous medical history, examination report, pregnancy status, history of allergies, and the help desired. As of October 2019, this study had obtained 5,411 pieces of structured case information.

All patients who asked questions were registered platform users and had signed an 
online service agreement with the platform. The informed consent was obtained from all the online patients.

2.2. Construction of a keyword lexicon of patients'self-reported rehabilitation care problems after hip replacement

The patients' self-reported language expressions were inconsistent and colloquial, and invalid corpus data, such as conjunctions and modal auxiliary words, would interfere with frequency analysis. Therefore, we first established a third-party lexicon to match the patients' corpus data, to classify and analyze them through machine learning.

\subsubsection{Setting primary and secondary labels}

According to a literature review, the results of previous qualitative interviews and the preliminary artificial corpus filter results, the classification of the patients' self-reported key problems fit well with the Omaha subsystem. Additionally, this study consolidated "sadness" in social psychology into mental health, changed "growth and development" to psychological growth, removed pregnancy and postpartum from physiological problems, and added other health-related behaviors to health-related behavior problems. After adjustments of the classifications, the criteria for classification of problems related to rehabilitation care after hip replacement in older individuals based on the Omaha system were established as four primary labels: environmental, psychosocial, physiological, and health-related behaviors, and 39 secondary labels (Figure 1). 


\section{Self-reported problems}

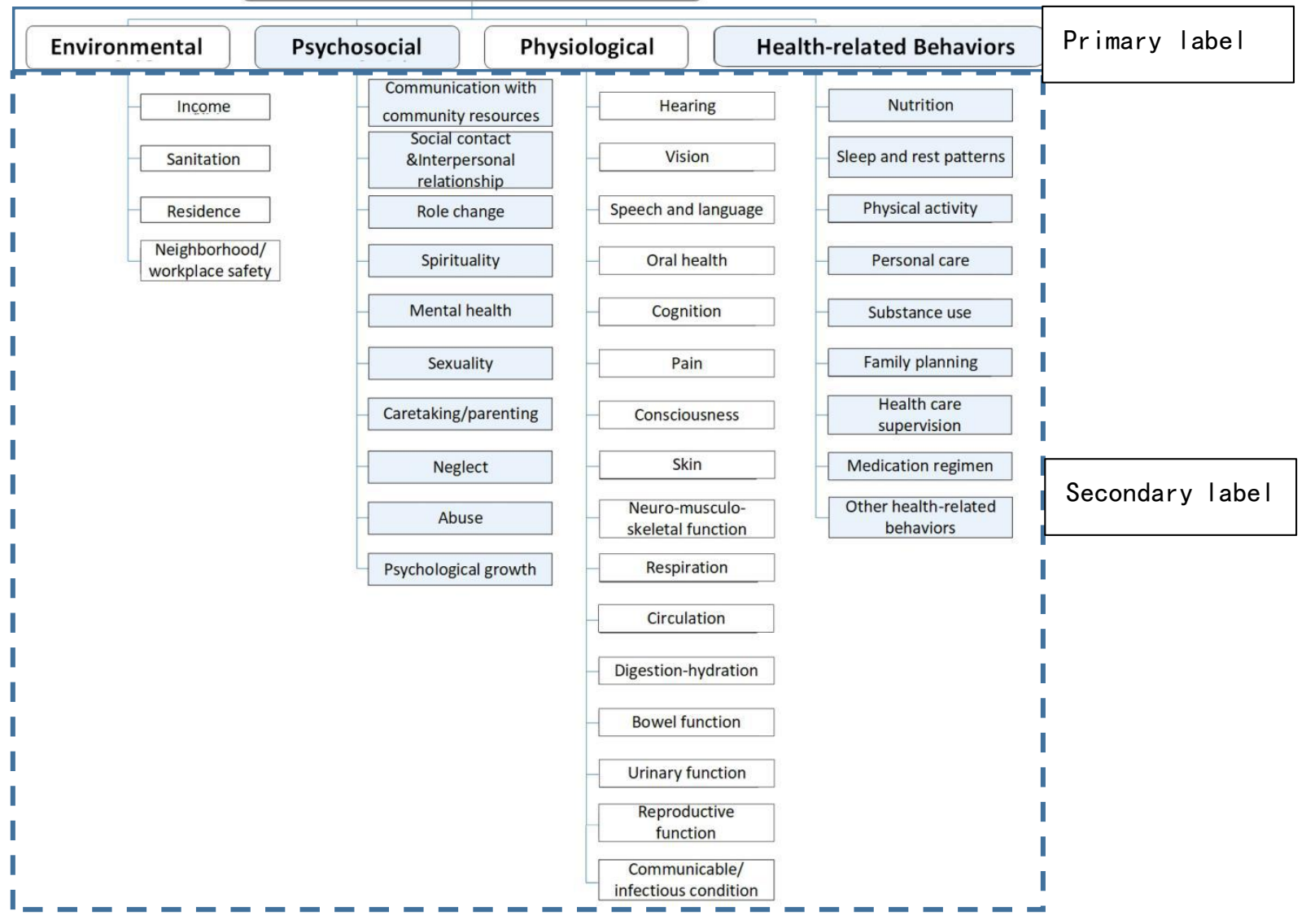

Figure 1. Classification criteria for self-reported problems in rehabilitation care after hip replacement in older patients, based on the Omaha classification system [8].

\subsubsection{Manual recognition and expert review classification}

Manual reading as well as classification and boxing were completed in April 2019 independently by the author and another orthopedic nurse with more than 10 years of experience with the 500 pieces of corpus data that had been extracted. Disagreements were adjudicated by a third person. In the preliminary filter, 332 patient keywords were identified and extracted, and were then classified and boxed according to the established primary and secondary labels using the Delphi method.

Twelve experts participated in this study. Ten experts met the following conditions: 1) 
Experts in medicine, nursing, or psychology engaged in bone and joint research, with rich scientific research experience, who had presided over or conducted research in related fields, and had a master's degree or above. 2) Medical staff engaged in clinical practice involving bone and joints, with more than 5 years of medical care or nursing experience, and with a bachelor's degree or above. The remaining two were computer and statistics experts, and provided guidance on the feasibility of corpus and data analysis. The mean self-evaluation score of the 10 experts for familiarity with the survey content was 0.87 (range: $0.5-1.0$ ), the judgment coefficient was $0.90(0.8-1.0)$, the comprehensive authority coefficient obtained was $0.89(0.65-1.0)$. The coordination coefficient for expert opinions in the two rounds of consultation was 0.427 and 0.413 , respectively, which was statistically significantly different by chi-square test.

\subsection{Keyword matching with supervised learning}

For supervised machine learning [11], natural language was processed, and machine processing was used to recognize human natural language. Supervised learning was used to match keywords with corresponding labels based on the constructed lexicon.

\subsubsection{Pre-processing and filtering}

As our study focused on problems of home-based rehabilitation care after hip replacement in older patients we filtered related health problems from the massive data. The conditional filter inclusion criteria, before matching, were as follows: Age $\geq 60$ years; hip replacement; first operation at the surgical site; home-based rehabilitation. The exclusion criteria were injuries at multiple sites; 
severe neurological diseases, such as stroke, that affected the activities of the limbs, comorbid diseases that seriously affected the muscles, soft tissues, and bones of the lower limbs, or immune-related rheumatic diseases that affected the activities of the limbs.

The number of historical samples after filtering according to the above inclusion and exclusion criteria was 485 .

\subsubsection{Keyword matching}

The regular matching method was used for batch processing of historical samples. Regular expressions can be used to determine whether a given string conformed to the filtering logic of regular expression (called "matching") [12], to obtain the required information from a string. Keywords that matched the constructed lexicon were extracted from the patients' self-reported language, providing a structured keyword sample set.We used the process shown in Figure 2 to complete the keyword matching.

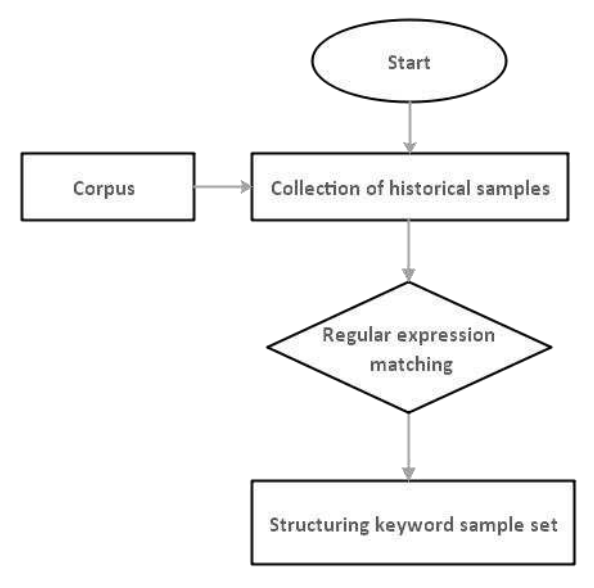

Figure 2. Structured data rearrangement model flowchart[12] 
Statistical analysis was conducted on the patients' health problems, and the frequency and ranking of primary and secondary labels in the matching labels determined. If self-reported keywords from the same patient appeared several times in the same level of a label dimension, this secondary label was counted as appearing once for this patient. For example, if a patient reported low income (keyword appearing for the first time) and low salary (keyword appearing for the second time), the algorithm determined that the patient had economic difficulties, and the frequency was recorded as 1 , both income and salary belonged to the same secondary label: economic condition.

\subsubsection{Calculation of correlation between labels}

We used the corr code of Python 3.6 to determine the correlation coefficient as an indicator of the degree of correlation between observation data, similar to Pearson's coefficient determination. The default was $\alpha=0.05$, with a confidence level of $95 \%$.

\section{Results}

\subsection{Summary of primary labels}

Patients reported 1,246 physiological problems, 673 health-related behavior problems, 425 psychological problems, and 127 environmental problems that were identified as home-based rehabilitation problems under the primary labels. The radar chart (Figure 3) shows that the majority of the patients' problems were physiological. 


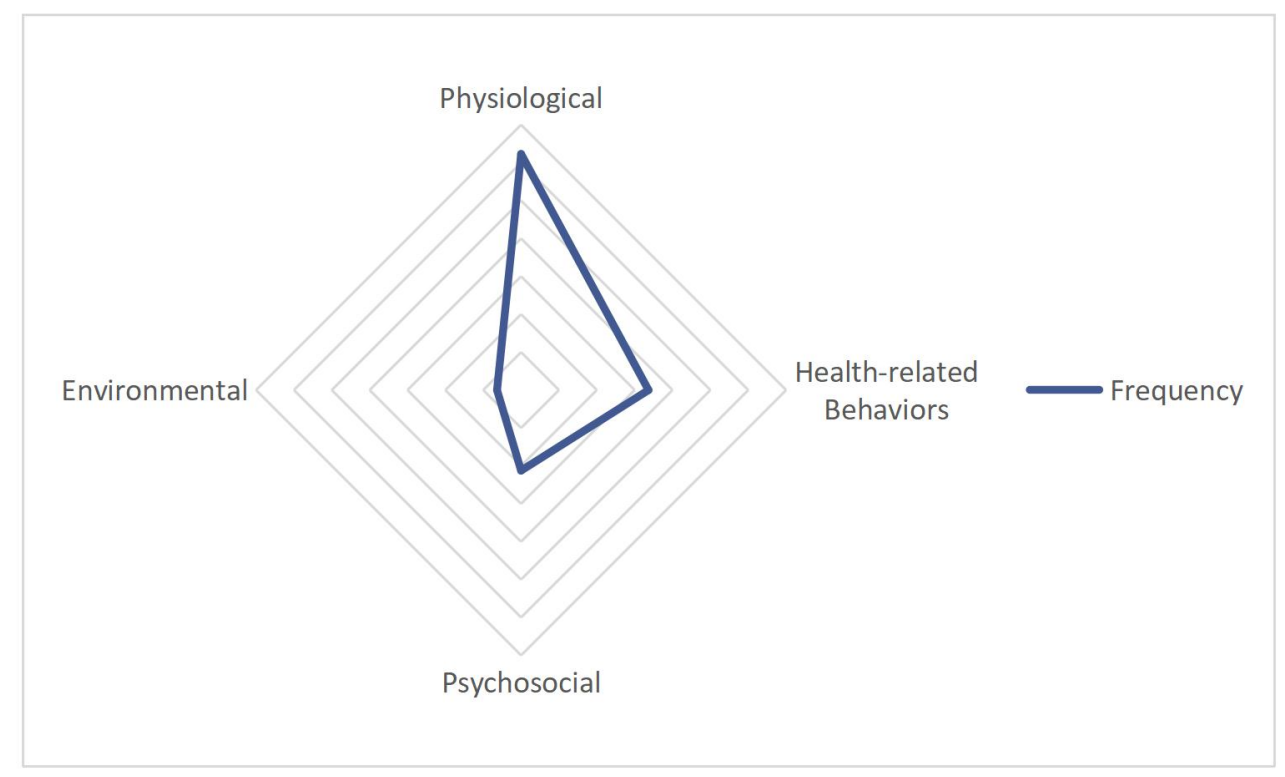

Figure 3. Radar chart of primary labels

\subsection{Summary of secondary labels}

As shown in Figure 4, problems related to neuromusculoskeletal function, interpersonal relationships, and pain were the top three secondary labels among patients' self-reported problems. The Pareto curve shows that more than $80 \%$ of the subjects' health-related problems consisted of issues related to neuromusculoskeletal function, interpersonal relationship, pain, health care supervision, physical activity, vision, nutrition, and residential environment. 


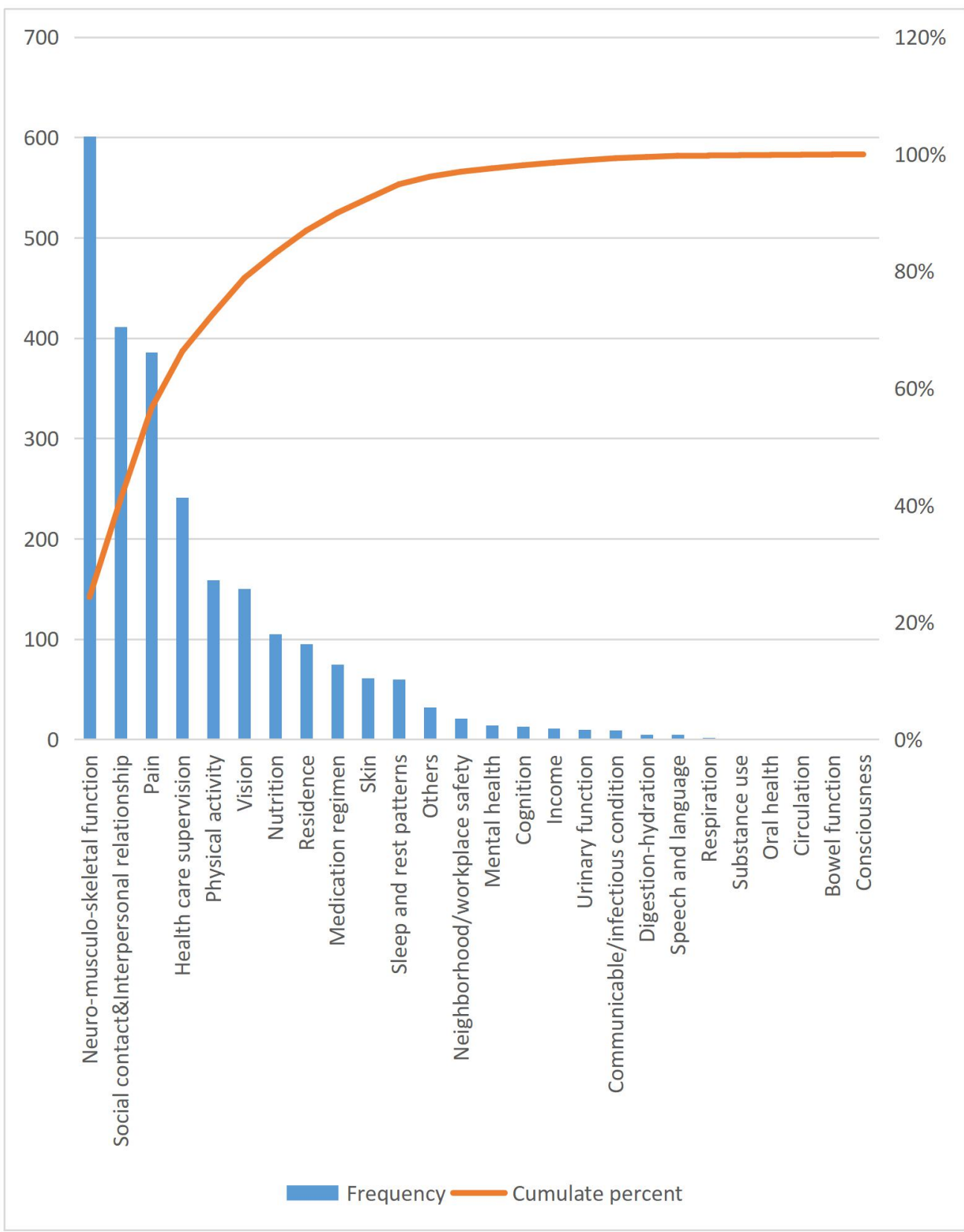

Figure 4. Pareto chart of secondary labels

\subsection{Subdivision ratios of each dimension}

As shown in Figure 5, physiology was the most frequently occurring primary label. Problems reported most by patients were related to neuromusculoskeletal function, which ranked first among the secondary labels. Interpersonal relationships was the main problem in the psycho-social dimension, and ranked second among all secondary labels. Additionally, the 
secondary label of health care guidance was the most frequent issue in the health-related behavior dimension. The secondary label of residential environment was the most frequent secondary label in the environment dimension.

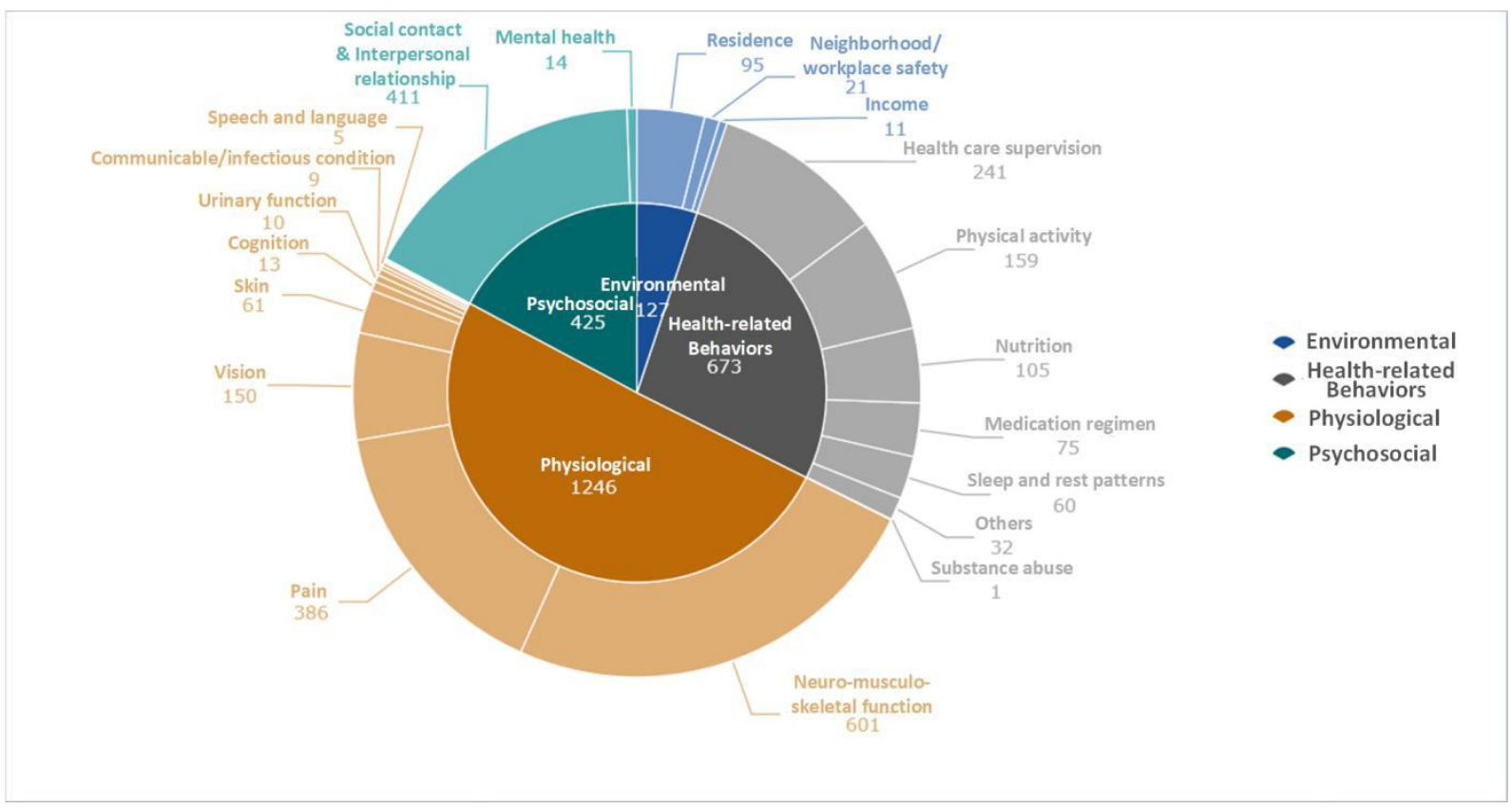

Figure 5. Tag summary pie charts for all labels

\subsection{Frequencies of patients' reported problems in different time periods}

As shown in Table 1, the frequency of problems reported by patients at different stages of rehabilitation differed. The stage with the highest frequency of self-reported problems was 6-months postoperatively. Patients at all stages mainly reported physical problems. Compared with other stages, the frequency of psycho-social problems was higher within 1 week; physiological problems were most prominent within 1 month, and health-related behaviors were most common after 1 month and within 6 months postoperatively. 
Table 1. Frequencies of problems reported by patients in different rehabilitation phases

\begin{tabular}{|c|c|c|c|c|}
\hline Primary label & $\begin{array}{l}\text { Within } 1 \\
\text { week }\end{array}$ & $\begin{array}{l}\text { Within } 1 \text { month } \\
\qquad(>1 \mathrm{w})\end{array}$ & 2-6 months & After 6 months \\
\hline Environmental & $6(5 \%)$ & $17(6 \%)$ & $27(5 \%)$ & $65(5 \%)$ \\
\hline Psychosocial & $24(20 \%)$ & $51(18 \%)$ & $81(14 \%)$ & $210(17 \%)$ \\
\hline Physiological & $59(50 \%)$ & $148(52 \%)$ & $288(50 \%)$ & $598(50 \%)$ \\
\hline \multicolumn{5}{|l|}{ Health-related } \\
\hline Behaviors & $29(25 \%)$ & $66(23 \%)$ & $180(31 \%)$ & $334(28 \%)$ \\
\hline Total & 118 & 282 & 576 & 1,207 \\
\hline
\end{tabular}

Data are given as frequency (percentage of current phase)

\subsection{Correlation analysis between dimensions}

This study used a non-questionnaire analysis, and deep mining of data interactions, etc. could only be performed manually on the captured data. Therefore, we used pairwise correlation analysis of all matching labels to explore the interactions between problems, and between problems and rehabilitation phases Using a significance level $\alpha=0.05$ and degree-of-freedom $n-2=485-2=483$, the tested statistics needed to be $>1.964887641$ to be significant. As the primary labels were all very weakly correlated, and each label was very weakly or not correlated to the rehabilitation phases, we only considered correlations between secondary labels with $\mathrm{r} \geq 0.2$. 
Table 2. Correlation analysis between secondary labels $(\alpha=0.05, \mathrm{n}=483)$

\begin{tabular}{|c|c|c|c|}
\hline Label A & Label B & $\begin{array}{l}\text { Correlation } \\
\text { coefficient }\end{array}$ & $\begin{array}{l}\text { Statistics } \\
\text { tested }\end{array}$ \\
\hline Speech and language & Consciousness & 0.45 & 10.93184132 \\
\hline Nutrition & Medication regimen & 0.40 & 9.542014593 \\
\hline Circulation & $\begin{array}{l}\text { communicable/infectious } \\
\text { condition }\end{array}$ & 0.33 & 7.6977035 \\
\hline Physical activity & Health care supervision & 0.33 & 7.671889236 \\
\hline Pain & Neuromusculoskeletal function & 0.29 & 6.709414938 \\
\hline Respiration & Other health-related behaviors & 0.24 & 5.48411013 \\
\hline Sleep and rest patterns & Physical activity & 0.23 & 5.222293589 \\
\hline $\begin{array}{l}\text { Neuromusculoskeletal } \\
\text { function }\end{array}$ & Physical activity & 0.23 & 5.187830114 \\
\hline Residence & Vision & 0.20 & 4.562193348 \\
\hline Sleep and rest patterns & Other health-related behaviors & 0.20 & 4.55268952 \\
\hline
\end{tabular}

\section{Discussion}

\subsection{Consistency with other research}

Based on our label analyses, the frequency of problems reported in the physiological dimension was the highest, particularly during the first month after surgery. Neuromusculoskeletal function issues ranked first among the secondary labels. The need for 
intervention in terms of neuromusculoskeletal function is a common problem after most orthopedic surgeries, and strength training can help patients achieve a better state of recovery. The actual physical function recovery of patients is reported to be very different from expectations, indicating the patient's need for intervention [13, 14]. Nursing staff should provide functional recovery and physiological guidance. Physiological problems may also coexist with other problems, such as psychological expectations, and overall analysis is required for comprehensive judgment and intervention.

In our study, the most frequently-occurring keyword self-reported by patients from 1 to 6 months post-operatively was pain. In previous qualitative research, we found that medical staff can recognize patients' need for pain control and provide a measure of attention. Relevant guidelines in China and other countries have reached consensus on the need for pain control after hip replacement [15]. However, as it involves medication compliance and standardization during home-based rehabilitation, many patients still do not achieve good pain control. Community hospitals and Grade A Class 3 hospitals need to establish postoperative pain medication standards. There is an increasing number of studies on compliance with home-based rehabilitation medication, but this needs to be considered specifically for older patients.

This study also analyzed the correlation between pain labels and other problems. Differences in pain related to neuromusculoskeletal function, physical activity, supervision of health care, sleep and rest patterns, nutrition, other health-related behaviors (such as integrated traditional Chinese and Western medicine treatment), and the intervention requirements for following the doctor's medication orders were statistically significant (Table 
2). This suggests that pain might co-occur with problems of function, activity, sleep and rest, and behavior, or may even interact with these problems. Chinese patients often use plasters, hot compresses, acupuncture (in hospital) and other health-related behaviors during the rehabilitation stage. We found that pain and such related behaviors were positively correlated.

\subsection{New problems identified}

\subsubsection{Chinese patients face marked problems with nutrition intervention}

In this qualitative big data analysis patients required marked nutritional intervention

(Figures 4). In the primary label dimension of health-related behaviors, nutrition-related problems ranked third. Moreover, as shown in the secondary label correlation analysis (Table 2), nutritional problems were weakly correlated with following doctors' medication orders ( $\mathrm{r}$ $=0.4)$. The reason for the prominence of these problems in older patients in China may be related to advanced age and culture. Compared to young people, older patients will experience different degrees of decline in various physical functions, such as reduced digestive function, changes in taste, and decreased metabolic capacity [16]. There is often an imbalance between nutrient supply and consumption, causing nutrition-related problems. Moreover, in Chinca, food is often used as medicine [17]. Patients often consult medical staff in terms of food choice during illness. Our health education for patient rehabilitation includes detailed guidance on nutrition, but only in terms of comprehensiveness and quantity from the perspective of Western medicine, while the types of food that should be selected needs to be determined jointly by medical staff. For medical staff, mastering Traditional Chinese Medicine(TCM) principles for health maintenance with food and diet therapy can provide 
patients with specific nutritional guidance and may be significant for medical practice.

\subsubsection{The importance of the neighborhood and workplace}

Neighborhood/workplace and residential environment keywords appeared 21 times and 95 times, respectively, in our analysis of related keywords. This frequency was much higher than that of infection problems, which typically concern medical staff. Previous studies showed that there is a gap between the patient's reported improvement in the home environment and the actual situation, which requires attention [18]. Residential environment and vision are correlated to a certain degree, which suggests that the vision problems reported by the patients in this study might be caused by the residential environment. Older patients face various problems in returning to society after home rehabilitation, given that their joint functions do not yet allow use of equipment in the home, neighborhood, and workplace. Society should jointly promote construction of residential environments and workplaces that cater to the needs of older people.

\subsubsection{Psychological problems urgently requiring guidance}

Patients relating their real-life problems is an "open-ended" narrative. We found that patients generally had less self-reports related to psychology. However, other "question-and-answer" studies that used questionnaires showed that older patients had a high level of related needs. Older hip replacement patients are more likely to have functional problems of the body, which affect their quality of life and leads to depression, anxiety, and other negative emotions. Depression is common in older hip fracture patients (prevalence rate of $9-47 \%$ ) [19]. Related emotions reduce the mobility of older patients, resulting in slow functional recovery. Older patients often feel inferior because they were hospitalized. In 
different stages of recovery, patients successively felt depression, melancholy, hopelessness, and fear for the future, which lasted for several months after discharge from the hospital, and even hampered their recovery [20]. This may be due to concerns about burdening the family, or due to Chinese culture, which is characterized by introversion and subtlety, or because patients conducting online consultation were more inclined to solve problems related to function and pain. Together, these factors led to less self-expression of negative emotions than that of other problems during open-end self-disclosure. Therefore, medical staff, particularly nursing staff closest to the patients, should guide patients in a timely manner, and actively intervene in psychological problems, thereby improving the mental health and interpersonal skills of older patients.

\subsection{The efficiency of big data algorithms}

Compared with other survey methods, such as qualitative research and traditional quantitative research, big data are characterized by volume (large), velocity (high speed), Variety (diversity), value (low value density), and veracity (authenticity) [6]. Due to the different ways of obtaining data, the use of artificial intelligence operations to capture and use big data is more efficient than other methods. After completing the code, more than 2,000 pieces of data were rapidly obtained in this study, while automatic data importing from traditional questionnaire surveys requires template design and entry of data in strict accordance with the specified format through a terminal. Thus, it is time-consuming and labor-intensive to collect questionnaire items on research related to home-based rehabilitation. The present study used the law of big data to obtain the maximum network data size possible, 
and artificial intelligence capture was also fully automated. Thus, efficiency was markedly improved compared with manual filling and data input.

\subsection{Study limitations}

Due to time and manpower limits, we could only perform preliminary filtering based on previous research and manual analysis of the corpus. The lexicon could be further improved in future. Deep learning can then be used with deep neural networks to solve the problem of feature expression. However, deep learning models require a large amount of training data. Due to time and resource limitations, the data currently mined are still insufficient for deep learning; further research will be needed. With the development of an authoritative standard lexicon, keyword classification will be unified, so that medical staff can more quickly identify the related health concerns of patients after hip replacement.

This study also attempted to build a correlation model between the rehabilitation phases and the patients' self-reported outcomes. However, due to the small sample number, an accurate model could not be obtained, and only fuzzy statistics could be produced. More patient data are required to obtain an accurate model.

Many older and advanced age patients are unable to surf the Internet and use smart phones, implying a certain bias in this study . However, the methods provided in this study provide a reference for future related evaluations involving the mining of online clinical medical data.

\section{Conclusions}


The evaluation method developed here may provide a basis for the rapid, economical, and comprehensive evaluation of patients, using existing massive data resources, and can facilitate formulation of suitable intervention strategies for patient rehabilitation care in future.

\section{Abbreviations}

TCM: Traditional Chinese Medicine

\section{Acknowledgements}

We would like to thank Editage (www.editage.cn) for English language editing.

\section{Author contributions}

CJ and HF handled the data collection process and writing. CJ, ZXX, and SY contributed during the process of formal analysis. CJ, QY, WQ, and SY were involved during methodology and project adminstration. HF provided major technical support and software operations. .WL and WQ revised the draft of the paper. All authors provided input into drafts and approved the final draft of the manuscript.

\section{Conflicts of interest}

The authors declare that they have no competing interests.

\section{Consent for publicationData statement Availability of data and materials}


Not applicable.

\section{Data statement Availability of data and materials}

The dataset supporting the conclusions of this article is available from the corresponding author upon reasonable request.

\section{Ethics approval and consent to participate}

Our study had been approved by the ethics committee of Ethics Committee of Shanghai Tenth People's Hospital (2016-RES-93). All methods were performed in accordance with the relevant guidelines and regulations. Informed consents had been obtained from all the patients.

\section{Funding}

NA.

\section{Author Details}

${ }^{1}$ Shanghai Tenth People's Hospital, School of Medicine, Tongji University, Shanghai, China, ${ }^{2}$ Smart Engineering Research Institute, Shanghai Investigation, Design \& Research Institute Co.,Ltd., Shanghai, China, ${ }^{3}$ Orthopaedics Department, Changhai Hospital, the Second Military Medical University, Shanghai, China, ${ }^{4}$ School of Medicine, Jinggangshan University, Ji An, China. 


\section{Summary table}

\section{What was already known on the topic}

- As the population ages, total hip replacement surgeries are increasingly performed.

- Due to the short length of hospitalization, rehabilitation care is mainly home-based.

- The needs and concerns about such home-based rehabilitation are constantly changing, and should be continuously evaluated.

\section{What this study added to our knowledge}

- The corpus of patients' self-reported rehabilitation care problems after hip replacement constructed based on the Omaha system in this study will provide a reference for the unified identification of issues related to hip replacement.

- The methods provided in this study provide a reference for future assessments of self-reported outcomes and intervention needs of patients. In a medical environment with limiting conditions, the mining of online clinical medical data can provide new ideas and evidence supporting precision care. 


\section{References}

[1] Wang HM, Paying attention to the health status of the older in China and promoting the national strategy of healthy aging,Chin. J. Epidemiol.Chinese journal of Epidemiology.2019, 40(3):259-65.doi: 10.3760/cma.j.issn.0254-6450.2019.03.002

[2] Curtis E , Litwic A, Cooper C , et al. Dennison, Determinants of muscle and bone aging, J. Cell. Physiol. 2015, 230(11):2618-25. https://doi.org/10.1002/jcp.25001

[3] Li N, Liu HN, Gong XF, Zhu SW, Wu XB, He L, Epidemiological analysis of hospitalized patients with femoral neck fracture in a first class hospital of Beijing. J. Peking Univ. Health Sci. 48 (2016) 292-6. https://doi.org/10.3969/j.issn.1671-167X.2016.02.021

[4] Hoogland J , Wijnen A, Munsterman T , et al. Feasibility and patient experience of a home-based rehabilitation program driven by a tablet app and mobility monitoring for patients after a total hip arthroplasty, JMIR Mhealth Uhealth, 2019, 7(1).e10342. https://doi.org/10.2196/10342

[5] Kingsbury SR , Dube B , Thomas CM , et al.Is a questionnaire and radiograph-based follow-up model for patients with primary hip and knee arthroplasty a viable alternative to traditional regular outpatient follow-up clinic?Bone Joint J. 2016, 98-B(2):201-8.https://doi.org/10.1302/0301-620X.98B2.36424

[6] Ristevski B, Chen M. Big Data Analytics in Medicine and Healthcare. J Integr Bioinform. 2018 May 10;15(3):20170030. https://doi.org/10.1515/jib-2017-0030

[7] Zhang L, Hall M, Bastola D. Utilizing Twitter data for analysis of chemotherapy. Int J Med Inform. 2018 Dec;120:92-100. https://doi.org/10.1016/j.ijmedinf.2018.10.002

[8] Martin KS, Norris J. The Omaha System: a model for describing practice. Holist Nurs 
Pract. 1996 Oct;11(1):75-83.https://doi.org/10.1097/00004650-199610000-00011

[9] Altiner M, Secginli S, Kang YJ. Refinement, reliability and validity of the Time Capture Tool (TimeCaT) using the Omaha System to support data capture for time motion studies. Jpn J Nurs Sci. 2020 Apr;17(2):e12296. https://doi.org/10.1111/jjns.12296

[10] Benke K, Benke G. Artificial Intelligence and Big Data in Public Health. Int J Environ Res Public Health. 2018 Dec 10;15(12):2796.https://doi.org/10.3390/ijerph15122796

[11] Tack C. Artificial intelligence and machine learning | applications in musculoskeletal $\begin{array}{lllll}\text { physiotherapy. } & \text { Musculoskelet } & \text { Sci } & \text { Pract. } & 2019\end{array}$ Feb;39:164-9.https://doi.org/10.1016/j.msksp.2018.11.012

[12] Chang Z,Lv Y, Mass data cleaning system based on regular expression. J. Comput. Appl. 2019,39(10):2942-7.doi:10.11772/j.issn.1001-9081.2019030492

[13] Peeters CM, Visser E, Van de Ree CL, Gosens T, Den Oudsten BL, De Vries J. Quality of life after hip fracture in the elderly: A systematic literature review. Injury. 2016 Jul;47(7):1369-82. https://doi.org/10.1016/j.injury.2016.04.018.

[14] Gustafsson BA, Ponzer S, Heikkilä K, Ekman SL. The lived body and the perioperative period in replacement surgery: older people's experiences. J Adv Nurs. 2007 Oct;60(1):20-8. https://doi.org/10.1111/j.1365-2648.2007.04372.x

[15] National Guideline Centre (UK). Evidence review for long-term follow-up and monitoring: Joint replacement (primary): hip, knee and shoulder: Evidence review T. London: National Institute for Health and Care Excellence (UK); 2020 Jun. PMID: 32881449.Available from:https://www.nice.org.uk/guidance/ng157 [16] Dong L, Xiao R, Cai C, Xu Z, Wang S, Pan L, Yuan L. Diet, lifestyle and cognitive 
function in old Chinese adults. Arch Gerontol Geriatr. 2016 Mar-Apr;63:36-42.

[17] Ju XR, On the thought of balanced diet in Chinese medicine, Jiangsu J. Trad. Chin. Med. 2000, 021(012):1-4.

[18] Xu HL, Cheng SZ, Yan FJ, Investigation and analysis of the home environment improvement needs of the older after hip fracture in Guangzhou, Mod. Clin. Nurs.2007(01):49-51.

[19] Holmes JD, House AO. Psychiatric illness in hip fracture. Age Ageing. 2000 Nov;29(6):537-46.

[20] Liu C, Shi L, He J, Zhang YN, et al.Adverse effect of depression on surgical outcome of artificial femoral head replacement in elderly patients. Chin. J. Joint Surg(Electronic Edition). 2020, 14(03):286-90. 


\section{Self-reported problems}

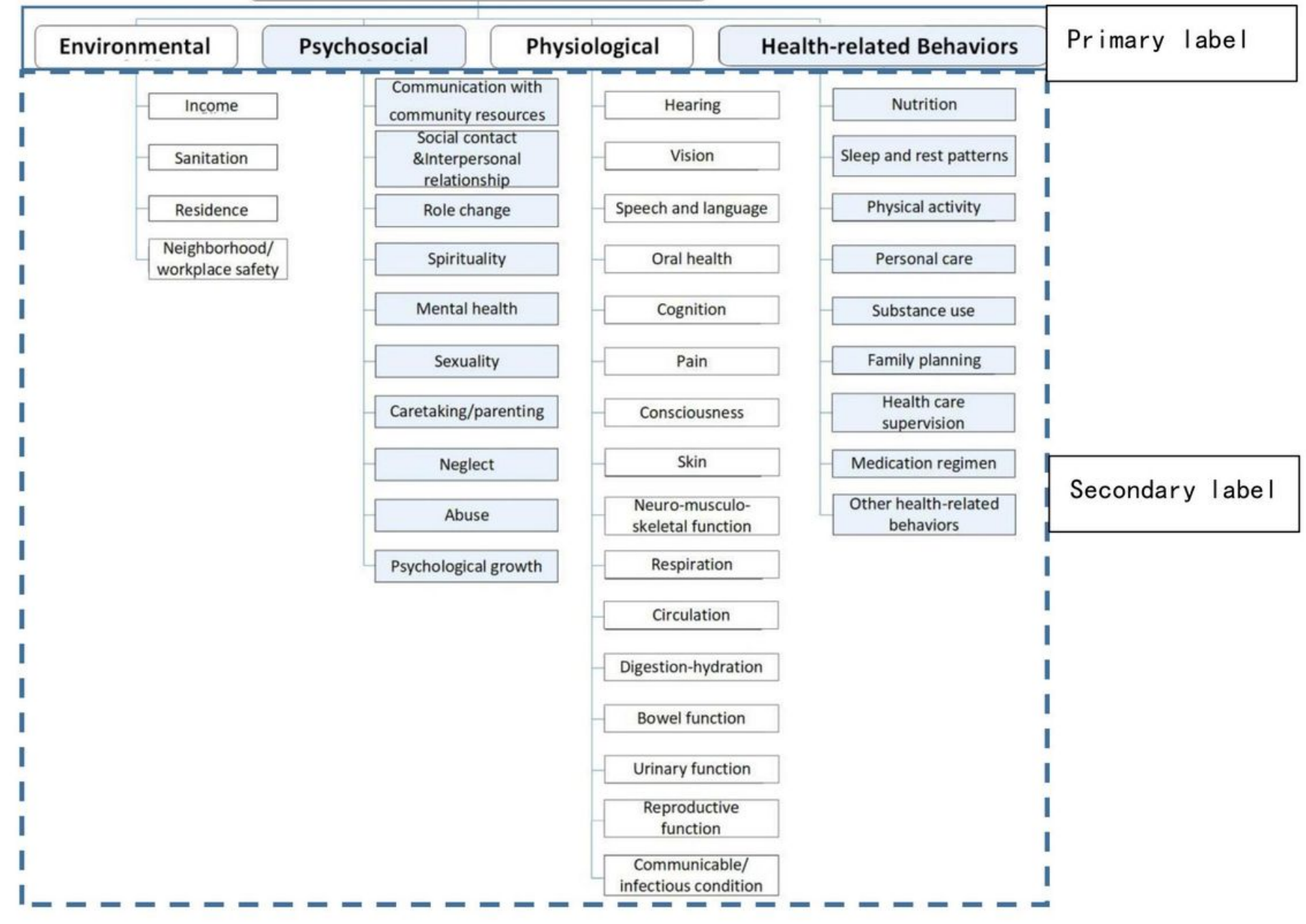

\section{Figure 1}

Classification criteria for self-reported problems in rehabilitation care after hip replacement in older patients, based on the Omaha classification system [8]. 


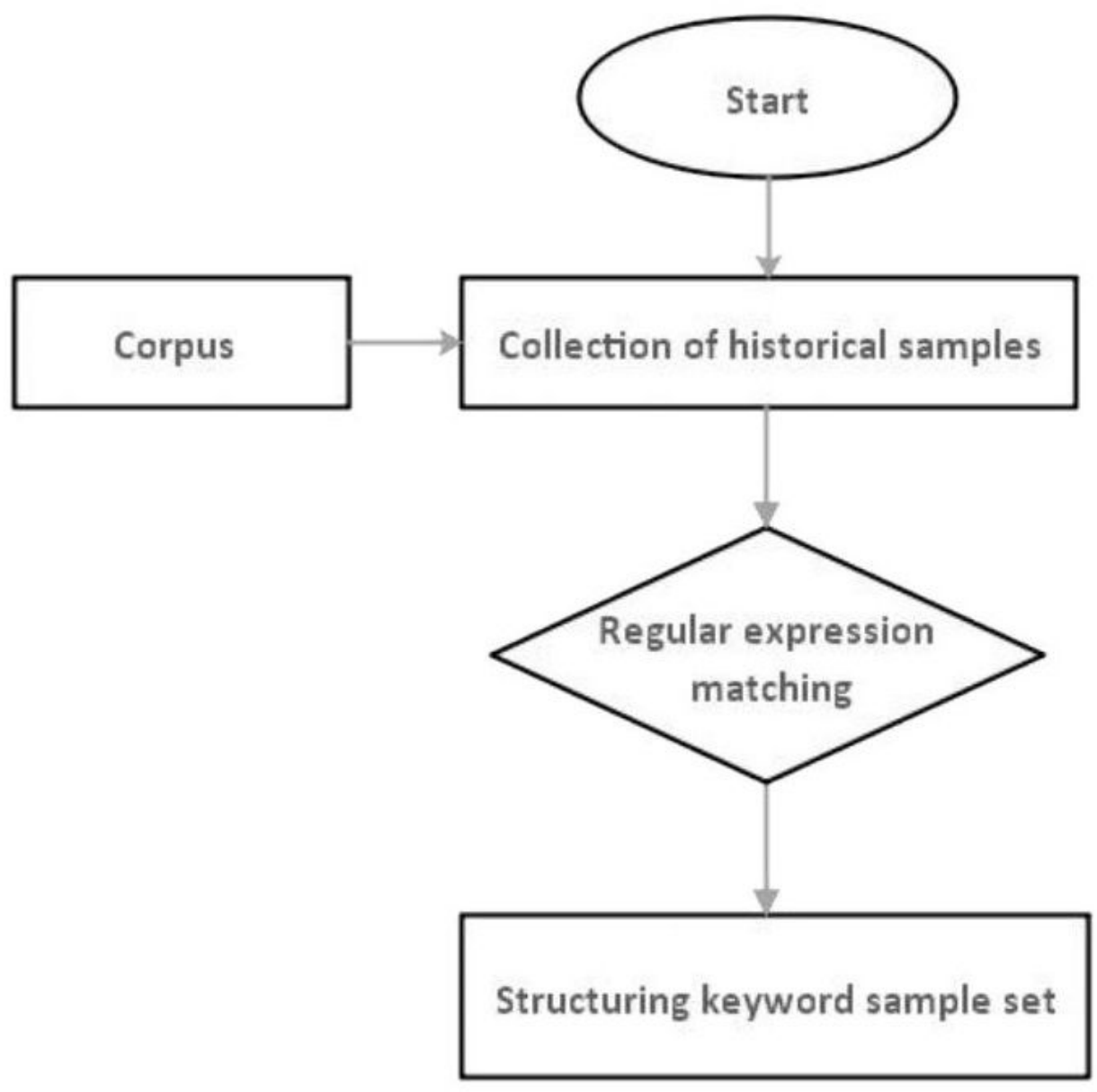

Figure 2

Structured data rearrangement model flowchart[12] 


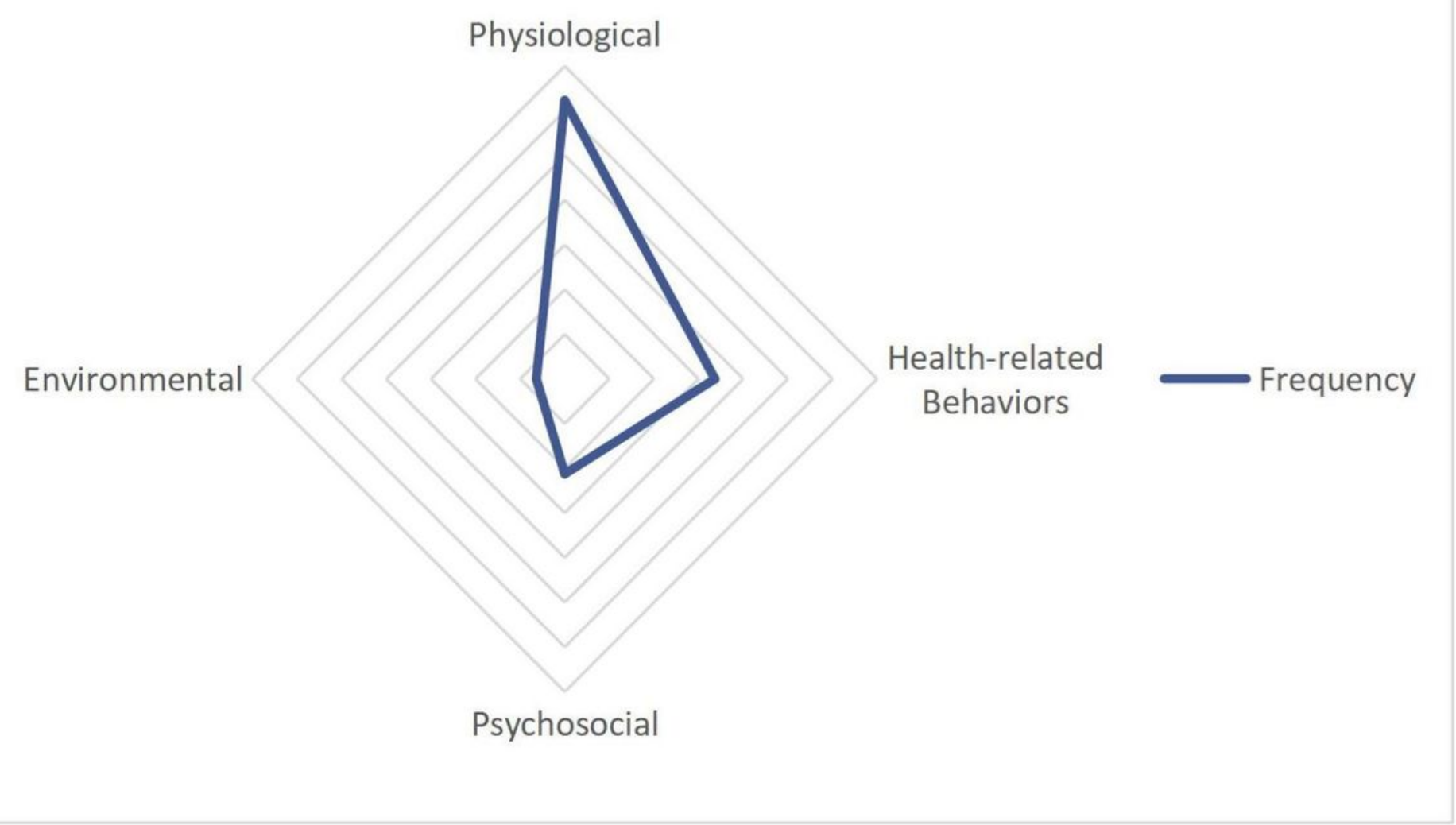

Figure 3

Radar chart of primary labels 


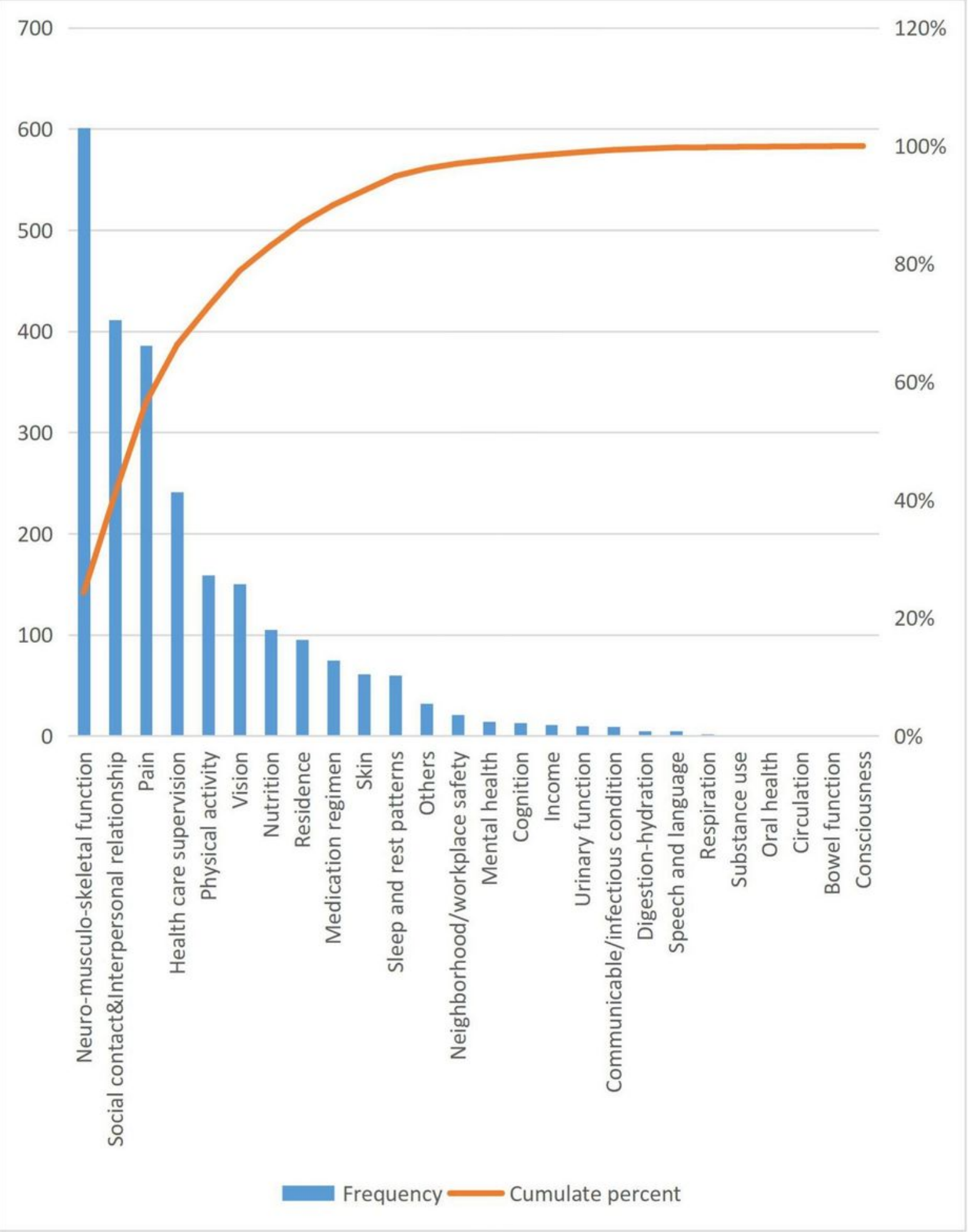

Figure 4

Pareto chart of secondary labels 


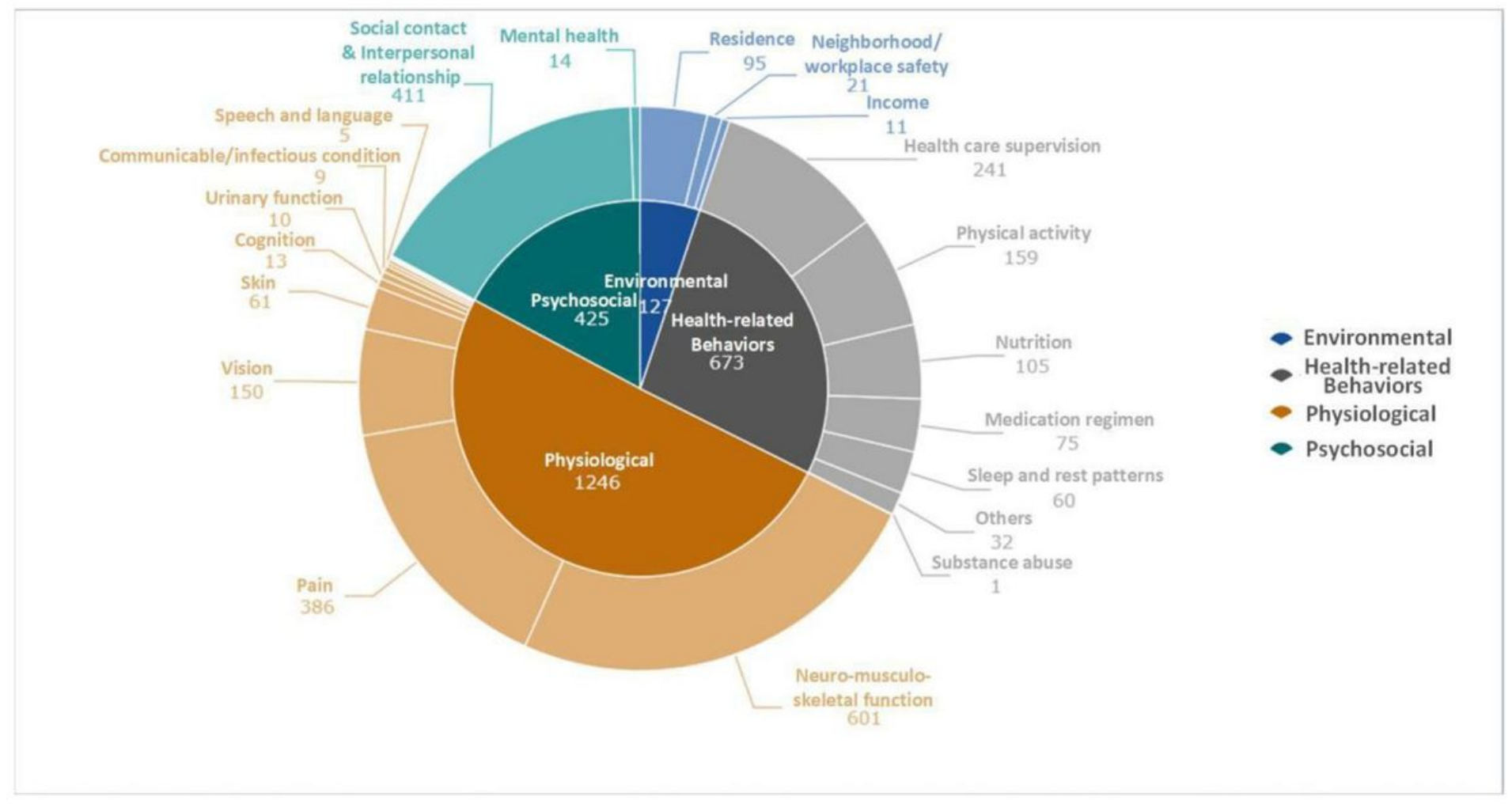

\section{Figure 5}

Tag summary pie charts for all labels 